\title{
Decreased lymphocytes and increased risk for infection are common in endogenous pediatric Cushing syndrome
}

\author{
Christina Tatsi ${ }^{1}$, Rebecca Boden ${ }^{1}$, Ninet Sinaii ${ }^{2}$, Meg Keil ${ }^{1}$, Charalampos Lyssikatos ${ }^{1}$, Elena Belyavskaya ${ }^{1}$, \\ Sergio D. Rosenzweig ${ }^{3}$, Constantine A. Stratakis ${ }^{1}$ and Maya B. Lodish ${ }^{1}$
}

BACKGROUND: Hypercortisolemia results in changes of the immune system and elevated infection risk, but data on the WBC changes in pediatric Cushing syndrome (CS) are not known. We describe the changes of the WBC lineages in pediatric endogenous hypercortisolemia, their associations with the markers of disease severity, and the presence of infections.

METHODS: We identified 197 children with endogenous CS. Clinical and biochemical data were recorded. Sixty-six children with similar age and gender, and normocortisolemia served as controls.

RESULTS: The absolute lymphocyte count of CS patients was significantly lower than that of controls, while the total WBC and the absolute neutrophil counts were significantly higher. These changes correlated with several markers of CS severity and improved after resolution of hypercortisolemia. Infections were identified in 35 patients (17.8\%), and their presence correlated to elevated serum morning cortisol, midnight cortisol, and urinary free cortisol levels, as well as with the decrease in absolute lymphocyte count.

CONCLUSIONS: Children with endogenous CS have abnormal WBC counts, which correlate with the severity of CS, and normalize after cure. Infections are common in this population; clinicians should be aware of this complication of CS and have low threshold in diagnosis and treating infections in CS.

$S^{i}$ ince 1948, when glucocorticoids (GCs) were first used for the management of rheumatoid arthritis, pharmacologic doses of glucocorticoids have been widely prescribed for their immunosuppressive properties (1,2). GCs currently constitute the first line therapy for many autoimmune, allergic, and malignant disorders, both in adults and in children (2).

GCs exert various effects on the immune system, affecting both the cellular components as well as the proteins of the inflammatory pathway (3). Specifically, GCs may alter the number of the white blood cells, resulting in leukocytosis, neutrophilia, lymphopenia, and eosinopenia as well as their function, leading to decreased activity of the natural killer cells (4-6). At the same time they also affect the concentration of the proinflammatory proteins, leading to the decrease of several proinflammatory cytokines, such as TNF-alpha, IL1, and IFN $\gamma$ (7).

Most of these effects have been reported in animal models or cell lines, while the human studies of the effects of GCs usually involve patients with additional underlying diseases. Endogenous Cushing syndrome (CS) may serve as a model of the net effects of GCs on the human body, and indeed adult patients with endogenous CS have been previously studied regarding the effects of hypercortisolemia on their immune system $(8,9)$. Endogenous CS leads to a significant increase in the WBC and the neutrophil counts, and decrease of the lymphocyte count (8). As a result, increased susceptibility to infections has been identified as one of the major morbidity and mortality factors in hypercortisolemic patients (9). The significance of this finding is highlighted in the endocrine society guidelines for the management of CS, where a recommendation for prophylactic antibiotic use in the adult patients with urinary free cortisol (UFC) levels greater than 5 fold the upper normal limit is included (10).

Despite these recommendations in adults with CS, no prior study has described the changes of the immune system in the pediatric CS population. We here aimed to describe these changes in a large cohort of pediatric patients with endogenous CS. We also investigated the presence of infections and their correlation with markers of severity of hypercortisolemia.

\section{METHODS}

\section{Subjects}

Through a retrospective chart review, we identified 197 patients diagnosed from 1998 until 2017 with endogenous CS before the age of 18 years old. The patients were evaluated at the Clinical Center of the National Institutes Health (NIH) under the protocols 97$\mathrm{CH}-0076,95-\mathrm{CH}-0059$ and 00-CH-0160. Our research was approved by the Eunice Kennedy Shriver National Institute of Child Health and Human Development (NICHD) Institutional Review Board. Written informed consent was obtained from the parents of the minor patients, and assent from the minor patients.

\footnotetext{
${ }^{1}$ Section on Endocrinology and Genetics, Eunice Kennedy Shriver National Institute of Child Health and Human Development (NICHD), National Institutes of Health, Bethesda, Maryland; ${ }^{2}$ Biostatistics and Clinical Epidemiology Service, $\mathrm{NIH}$ Clinical Center, Bethesda, Maryland; ${ }^{3}$ Immunology Service, Department of Laboratory Medicine, Clinical Center, $\mathrm{NIH}$, Bethesda, Maryland. Correspondence: M.B. Lodish, lodishma@nih.gov

Received 10 August 2017; accepted 5 October 2017; advance online publication 6 December 2017. doi:10.1038/pr.2017.278
} 


\section{Articles | Tatsi et al.}

The diagnosis of CS and the classification of the various etiologies was based on previously described criteria (11). The diagnosis was confirmed histologically after surgery for all patients that underwent surgical resection. For two of the patients with Cushing disease (CD), who underwent surgical treatment at an outside institution, review of the pathology report confirmed the presence of a corticotroph adenoma. Of the 38 patients with CS due to adrenal causes, 17 patients had confirmed clinical and genetic diagnosis of Carney Complex syndrome, while of the patients with $\mathrm{CD}$, two patients had Multiple Endocrine Neoplasia (MEN) syndrome type 1, and one patient had tuberous sclerosis.

Sixty-six children, with similar age and gender distribution as our patient cohort, who were referred to our institution for evaluation of possible hypercortisolemia and were found to have normal cortisol

Table 1. Clinical and biochemical characteristics of pediatric patients with CS

\begin{tabular}{lr}
\hline Characteristics & Value $\mathbf{N}=19$ \\
\hline Mean age at diagnosis, years $( \pm \mathrm{SD})$ & $12.5( \pm 3.4)$ \\
Mean duration of disease, years $( \pm \mathrm{SD})$ & $2.6( \pm 1.8)$ \\
Gender & \\
Male (\%) & $88(44.7)$ \\
Female (\%) & $109(55.3)$ \\
& \\
Race & \\
Asian (\%) & $9(4.6)$ \\
Black or African American (\%) & $14(7.1)$ \\
Multiracial or Other (\%) & $5(2.5)$ \\
White (\%) & $142(72.1)$ \\
Unknown (\%) & $27(13.7)$
\end{tabular}

Ethnicity

Hispanic or Latino (\%)

Not Hispanic or Latino (\%)

$155(78.7)$

Unknown Ethnic Group (\%)

$3(1.5)$

\section{At diagnosis}

Mean morning Cortisol level, mcg/dL ( \pm SD)

Mean midnight Cortisol level, mcg/dL ( \pm SD)

$18.6( \pm 13.2)$

UFC, $\mathrm{mcg} / 24 \mathrm{H}( \pm \mathrm{SD})$

$399( \pm 1465.1)$

\section{Etiology of CS}

Cushing Disease (\%)

$154(78.2)$

Ectopic ACTH production (\%)

ACTH-independent CS (\%)

\section{Postoperative course}

Cure (\%)

No cure after surgery (\%)

Recurrence (\%)

$13(6.6)$

No follow-up (\%) secretion, served as the control group. Most of these patients were obese, while some of them had additional pathologic disorders identified: diabetes insipidus $(n=1)$, connexin 26 defect and deafness $(n=1)$, fragile $\mathrm{X}(n=1)$, hypothyroidism, on replacement therapy and biochemically euthyroid at the time of the test, $(n=2)$, MEN syndrome type $1 \quad(n=1)$, developmental delay and learning difficulties $(n=2)$, Kallman syndrome $(n=1)$, precocious puberty $(n=1)$, and premature adrenarche $(n=1)$.

Demographic (age, gender, duration of disease), clinical (operation notes, pathology reports, presence of infection), hematology and biochemical data (CBC with differential, serum and urine cortisol, plasma $\mathrm{ACTH}$ ) were retrieved from the electronic medical record system. The first CBC with differential at the time of diagnosis was used for further analysis. The results from the CBC with differential done at the follow up visit closest to 1 year after treatment were used for the analysis after cure (mean time to follow up visit $14.1 \pm 11.6$ months). Serum cortisol and UFC results were documented for all the patients at the follow up visit to document the resolution of hypercortisolemia.

The average of the first three urinary free cortisol levels was calculated and is referred to as UFC. Urinary samples that were collected on days of additional tests, such as dexamethasone suppression test, were excluded. Morning cortisol refers to the average value of the 7:30am and 8:00am samples of serum cortisol, while midnight cortisol refers to the average value of the serum cortisol at 11:30pm and 12:00am. Plasma ACTH refers to the average value of 7:30am and 8:00am levels if available, or the plasma ACTH level collected closer to 0800 hours.

\section{CBC, serum and urine markers of CS}

CBC with differential was measured using the Sysmex XN 3000 automated hematology system (Lincolnshire, IL). The serum cortisol was measured via Solid-phase, competitive chemiluminescent enzyme immunoassay on Siemens Immulite 2500 analyzer, (Malvern, PA). UFC (average of the first three samples collected after admission) was measured in 24-h urine collections via High Performance Liquid Chromatography/ Tandem Mass Spectrometry (LC-MS/MS). The plasma ACTH was measured via chemiluminescent enzyme immunoassay on Siemens Immulite 200 XPi analyzer (Malvern, PA).

\section{Statistical analysis}

The results are presented as frequencies and percentages, and mean $( \pm S D)$. Data were assessed for distributional normality, and nonparametric tests were used for non-normally distributed data. Continuous data were compared between groups (e.g., CS vs. controls; CS with infection vs. CS without infection) using the twosample $t$-test or Wilcoxon rank-sum test, as applicable; paired continuous data (e.g., diagnosis vs. cure) were compared using the one-sample t-test or Wilcoxon signed-rank test. Categorical data between groups were compared by means of the $\chi^{2}$ or Fisher's exact tests, as appropriate, and paired data were compared using the McNemar's test. Correlation analyses utilized the Spearman's rank correlation coefficient. Data were considered statistically significant if the resulting $P$ value was $<0.05$. Analyses were carried out using SAS v9.4 (SAS Institute, Inc, Cary, NC).

\section{RESULTS}

\section{Characteristics of the patients}

The clinical and biochemical characteristics of the patients are shown in Table $\mathbf{1}$. The mean age at presentation was 12.5 years, with a range from 2.7 to 18 years of age. There were more female than male patients $(n=109,55.3 \%)$. In the control group, there were 66 individuals, with mean age of 12.5 years (range 2.9 to 17.9 years), and 37 (56\%) females. The mean duration of disease was $2.6 \pm 1.8$ years. At diagnosis, the 
mean morning serum cortisol level was $20.4 \pm 13.5 \mathrm{mcg} / \mathrm{dL}$, the mean midnight serum cortisol level was $18.6 \pm 13.2 \mathrm{mcg} /$ $\mathrm{dL}$, and the mean UFC level was $399 \pm 1465 \mathrm{mcg} / 24 \mathrm{H}$. Most of the patients were diagnosed with CD $(n=154,78.2 \%)$, while 38 patients (19.3\%) had ACTH-independent CS due to adrenal causes, and 5 patients $(2.5 \%)$ were diagnosed with CS due to ectopic ACTH production, which is consistent with the previously described relative frequency of the various etiologies of CS in children.

All the patients with CD underwent transphenoidal resection (TSS), while the patients with adrenal dependent CS underwent unilateral $(n=10)$ or bilateral $(n=28)$ adrenalectomy according to their underlying disease. Of the 5 patients with ectopic CS, previously reported in a separate case series (12), all patients underwent resection of the source of ectopic ACTH/CRH production (bronchogenic carcinoma $=1$, metastatic pancreatic neuroendocrine tumor $=2$, thymic carcinoid $=1$, metastatic hepatic neuroendocrine tumor $=1$ ), while additional therapeutic interventions were implemented depending on the persistence of their disease. Of the patients who were followed after surgery $(n=163,82.7 \%)$, 142 patients $(87.1 \%)$ had persistent remission of CS at their latest follow up, 13 patients $(8.0 \%)$ had recurrence of CS after initial remission, and 8 patients $(4.9 \%)$ were not cured after surgery, resulting in a total of 21 patients (12.9\%) with persistent or recurrent hypercortisolemia during any follow up (Figure 1).

\section{Comparison of the total WBC count and the various cellular components of the immune system between the patients with $\mathrm{CS}$ and the control group}

The total WBC count and the count of the various WBC types of the children with CS were significantly different from the corresponding values of the control group. The patients with CS had significantly lower absolute lymphocyte count $(2.19 \pm 0.78 \mathrm{~K} / \mathrm{mcL})$ compared to the control group $(2.55 \pm 0.84 \mathrm{~K} / \mathrm{mcL}, P=0.002)$. Additionally, they had significantly higher total white blood cell count $(9.64 \pm 2.84 \mathrm{~K} /$ mcL vs. $7.62 \pm 1.78 \mathrm{~K} / \mathrm{mcL}, P<0.001)$ and absolute neutrophil

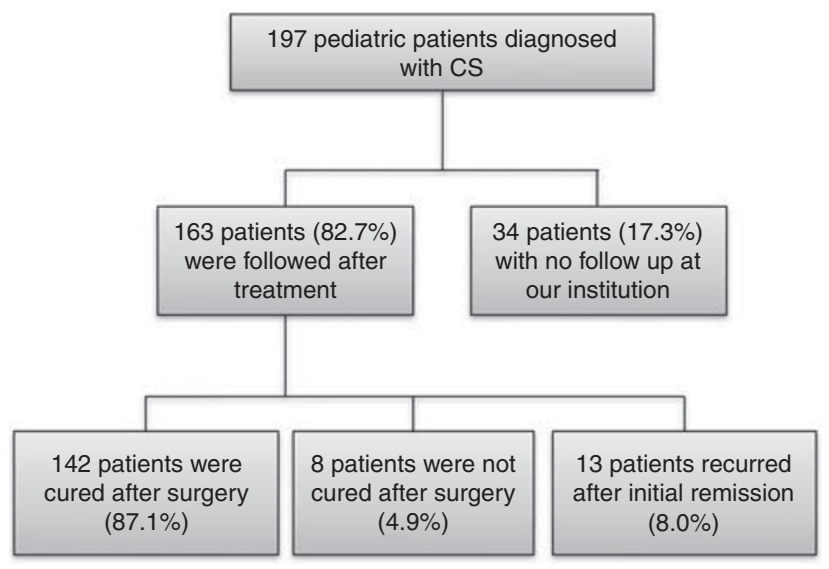

Figure 1. Flowchart of the disease course of pediatric patients with CS. count $(6.42 \pm 2.57 \mathrm{~K} / \mathrm{mcL}, \pm 2.57$, vs. $4.22 \pm 1.43, P<0.001)$ compared to the control group. When taking into account the age and gender specific normative cutoff values, $6.1 \%$ of our patients were lymphopenic at diagnosis compared to $1.5 \%$ of the control group (not statistically significant, $P=0.19$ ), while leukocytosis and neutrophilia were more prevalent in the CS population ( $45.7 \%$ and $34.7 \%$, respectively) compared to the control population $(18.2 \%$ and $9.1 \%$, respectively, $P<0.001$ for both).

\section{Comparison of cellular components of the immune system before and after resolution of hypercortisolemia}

For the patients who were followed after surgery $(n=163)$, total WBC count was available for 136 patients while complete CBC with differential was available for 126 of them. Comparison of the WBC count and the count of the various WBC types before treatment and after resolution of hypercortisolemia revealed significant improvement of all the WBC lineages. Specifically, the absolute lymphocyte count increased significantly from $2.15 \pm 0.78 \mathrm{~K} / \mathrm{mcL}$ at diagnosis to $2.88 \pm 1.04 \mathrm{~K} / \mathrm{mcL}$ after cure $(P<0.001)$. The total white blood cell count decreased from $9.31 \pm 2.55 \mathrm{~K} / \mathrm{mcL}$ at diagnosis to $6.83 \pm 1.88 \mathrm{~K} / \mathrm{mcL}$ after cure $(P<0.001)$, while the absolute neutrophil count decreased from $6.16 \pm 2.34 \mathrm{~K} /$ $\mathrm{mcL}$ at diagnosis to $3.25 \pm 1.5 \mathrm{~K} / \mathrm{mcL}$ after cure $(P<0.001)$ (Table 2). When taking into account the age and gender specific normative cut-off values, most patients with abnormal counts at diagnosis subsequently normalized at their follow-up visit $(90.0 \%$ with previous lymphopenia $(P=0.035)$, $91.1 \%$ with leukocytosis $(P<0.001)$, and $86.8 \%$ with neutrophilia $(P<0.001)$ normalized). When studying specifically the patients who had persistent or recurrent disease, the various counts showed temporary improvements at the period of remission of CS, with recurrence of several of the changes upon return of hypercortisolemia, as described in Table 2.

Correlation of the WBC changes with the markers of CS severity Several makers of Cushing severity, including the urinary free cortisol (UFC), the morning serum cortisol and the midnight serum cortisol, correlated with alterations of the WBC and the WBC types at diagnosis. Specifically, the absolute lymphocyte count was negatively correlated with the morning and midnight cortisol levels $\left(r_{\mathrm{s}}=-0.21, P=0.004\right.$ and $r_{\mathrm{s}}=-0.18$, $P=0.011$, respectively) and the UFC $\left(r_{\mathrm{s}}=-0.16, P=0.026\right)$. Conversely, the absolute neutrophil and the total WBC counts were positively correlated with some of the above markers (Figure 2). When investigating specifically the patients with $\mathrm{CD}$, the level of morning plasma ACTH was not related to immune cell counts $(P>0.05$ in all correlation analyses with the total WBC, absolute lymphocyte, and absolute neutrophil count). Although there were few patients with significantly elevated cortisol levels that could potentially influence the statistical analyses, the results remained significant for all the above analyses after excluding them. 


\section{Presence of infections in children with endogenous CS}

In order to assess the clinically significant outcome of the above changes, we identified the patients who had reported a significant infection (prolonged duration, persistent after one course of treatment, or opportunistic infections) within the 6 months prior to diagnosis, or perioperatively. We identified 35 patients $(17.8 \%)$ who fulfilled the above criterion. Most of the infections were fungal infections of the skin and mucosa. Specifically, 24/35 patients had evidence or history of prolonged fungal infections (tinea corporis, tinea capitis, tinea pedis, or vaginal candidiasis). In addition, bacterial and viral skin infections, sinusitis, urinary tract, IV line, and GI infections were also reported. Notably, we did not identify any opportunistic infections or any case of sepsis in our cohort.

\section{Correlation of WBC changes with infections and severity of CS}

There were remarkable differences between the group of CS patients with a concurrent infection and the remaining patients with CS. Specifically regarding the $\mathrm{CBC}$ changes, the absolute lymphocyte count was significantly lower in the group of patients with infection $(2.01 \pm 0.93 \mathrm{~K} / \mathrm{mcL})$ compared to those without $(2.24 \pm 0.74 \mathrm{~K} / \mathrm{mcL}, \quad P=0.047)$. Additionally, the patients with infection had significantly higher serum morning cortisol $(24.4 \pm 13.9 \mathrm{mcg} / \mathrm{dL}$, vs. $19.6 \pm 13.3 \mathrm{mcg} / \mathrm{dL}, \quad P=0.008), \quad$ midnight cortisol $(23.3 \pm 14.7 \mathrm{mcg} / \mathrm{dL}$, vs. $17.6 \pm 12.6 \mathrm{mcg} / \mathrm{dL} P=0.005)$, and UFC levels $(426 \pm 487.5 \mathrm{mcg} / 24 \mathrm{H}$, vs. $393 \pm 1603.4 \mathrm{mcg} / 24 \mathrm{H}$, $P=0.009$; Figure 3).

\section{DISCUSSION}

We report for the first time that children with endogenous hypercortisolemia have significant changes in the cells of their immune system, including decrease of the absolute lymphocyte count and increase of the total WBC and the absolute neutrophil count, which correlate with the serum and urine cortisol levels. The WBC count changes normalize after the resolution of hypercortisolemia, and return upon recurrence of the CS. We also demonstrate that the presence of infections, as the clinically significant outcome of these changes of the immune system, is significantly associated with the changes of the WBC counts and the indices of severity of CS.

Children with endogenous CS may be considered as the human model of the net effect of pharmacologic doses of GCs on the immune system. Several of the previous studies have described these effects in patients receiving GCs secondary to an underlying disease, such as an autoimmune, malignant, or atopic disorder (13-16). However, these patients may already have a dysregulation of their immune system and are frequently on multiple other therapeutic agents. Furthermore, the alterations of the immune system in endogenous CS have only been studied in the adult population, where additional age related illnesses may often be present $(8,17-19)$. On the contrary, children with endogenous hypercortisolemia, rarely have other comorbidities; thus, it is highly possible that the identified changes are the direct effect of the glucocorticoid 

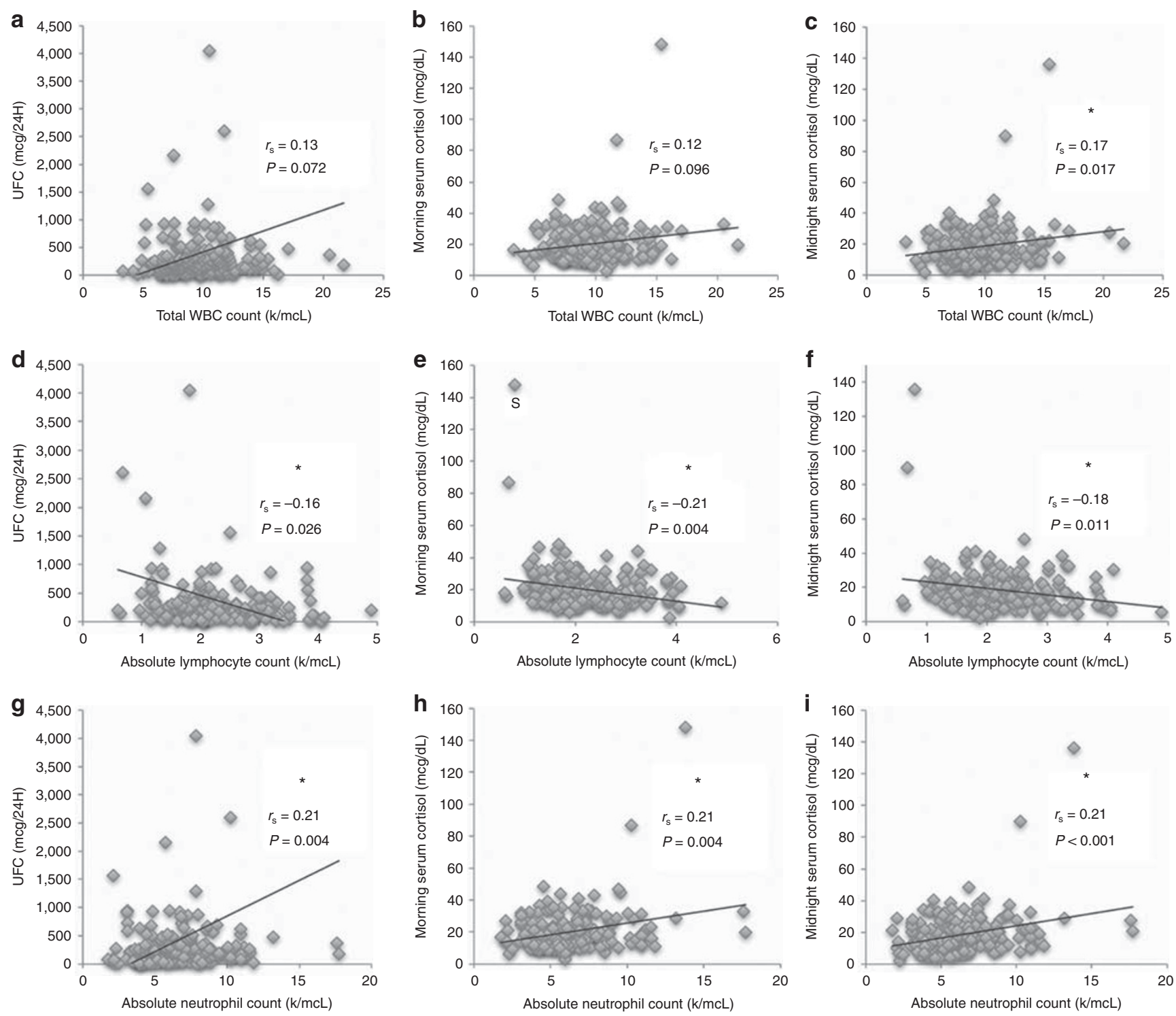

Figure 2. Scatterplots of the total WBC (a-c), absolute lymphocyte (d-f), and absolute neutrophil count (g-i), and their correlations with UFC (a,d,g), morning serum cortisol $(\mathbf{b}, \mathbf{e}, \mathbf{h})$, and midnight serum cortisol $(\mathbf{c}, \mathbf{f}, \mathbf{i})$ levels. Asterisk $\left(^{*}\right)$ indicates $P<0.05$.

excess. Children also exhibit continuous growth with increased plasticity, and the information that we get from diseases that present in this age could provide indirect insight on the adaptation of their immune system.

Given the high prevalence of childhood obesity, where almost one third of children and adolescents currently fall under the category of overweight or obese, it is of utmost importance to emphasize the signs that can be used by physicians to distinguish those individuals who require further workup for CS (20). One of the main findings of pediatric CS is the evidence of weight gain with concomitant height deceleration, which is not present in growing obese patient where height acceleration and tall stature are usually observed $(21,22)$. However, other typical findings of CS, such as striae, hirsutism, acne, acanthosis nigricans, myopathy, hyperglycemia, hyperlipidemia, and decreased bone mineral density, can sometimes be also seen in obese patients or they develop over a prolonged period of hypercortisolemia, when complications have already occurred or treatment becomes challenging (11,23-25). Although we cannot conclude anything about the timing of the $\mathrm{CBC}$ changes from our study (since the $\mathrm{CBC}$ we analyzed here was obtained after an estimated mean duration of disease of 2.6 years), it has been previously described that the changes of the WBC counts occur soon after the administration of GCs $(15,26,27)$. Thus $\mathrm{CBC}$ with differential, which is a low cost, minimally invasive and easy to interpret test, could potentially serve as a screening tool for CS in this population, although in the setting of an infection this should be interpreted in the context of additional clinical signs. A new study ought to look at the timing of this change and see if this test could serve for ruling in or out CS among children with obesity. 


\section{Articles | Tatsi et al.}
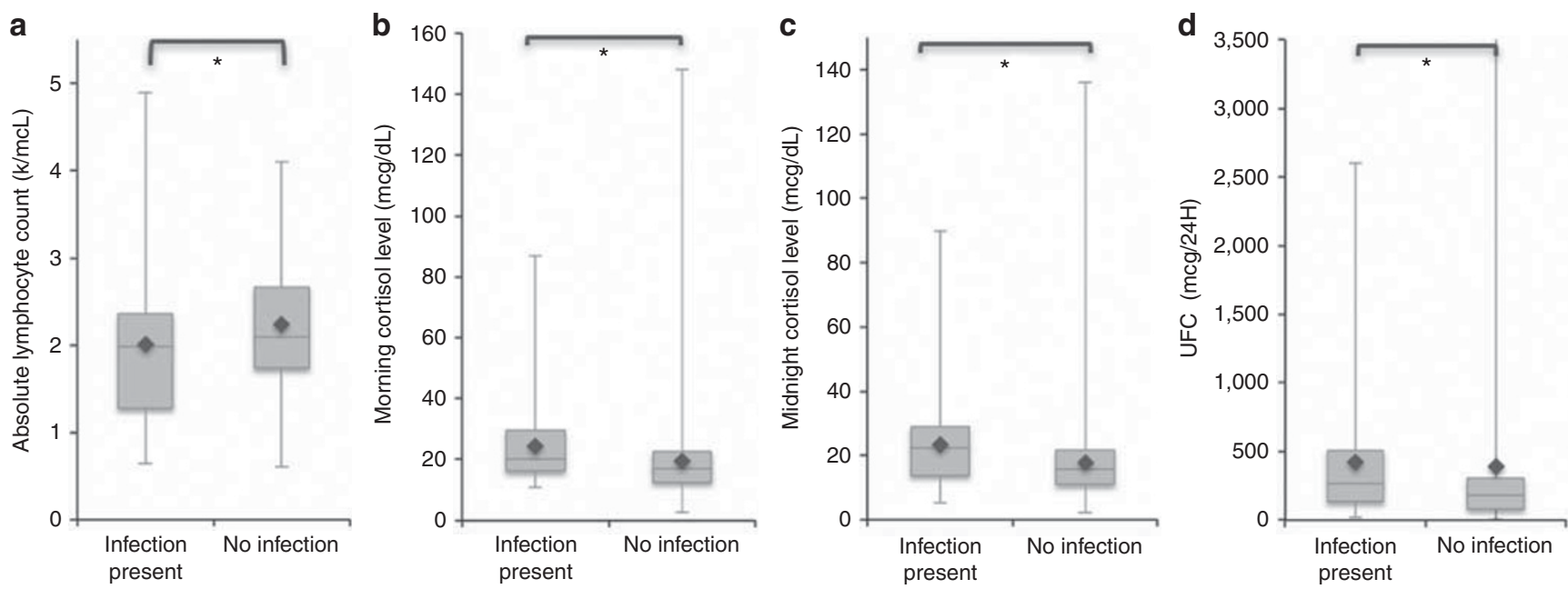

Figure 3. Boxplot diagrams of the absolute lymphocyte count (a), morning serum cortisol (b), midnight serum cortisol (c) and UFC level (d) of the patients with infection compared to those without. All values were significantly different $(P<0.05)$ between the two groups $\left(^{*}\right)$.

Additionally, the presence of a persistent or difficult to treat infection should prompt further workup for hypercortisolemia in suspicious patients. It is described in the literature that CS is associated with increased risk of infections (28). In a metanalysis by Stuck et al, (29) administration of GCs resulted in increased risk of infections in a dose dependent pattern. Increased infection risk has been also identified as a feature of adults with endogenous CS $(30,31)$. In the pediatric population, we confirmed the high frequency of infections; thus, the presence of an abnormal CBC, with low lymphocytes and high neutrophils, and the identification of a persistent infection in an obese child should indicate the need for further evaluation.

However, the majority of the infections in our cohort were mild, mostly fungal skin or mucosal infections, while invasive or opportunistic infections were not noted to have occurred. As we described, the presence of infection is positively associated with the serum and urinary cortisol levels. Given the rarity of ectopic CS in childhood, which is known to result in significant hypercortisolemia, the lower expected blood and urine levels of cortisol could potentially explain the milder nature of the reported infections. This is in accordance with the adult population studies, where many of the severe infections have been described in patients with ectopic/ paraneoplastic CS (9,31-33). However, this cannot exclude the possibility of a severe infection even in the pediatric population. Indeed, as previously reported by Gkourogianni et al (34), pediatric CS is a potentially fatal condition. Of the four deaths reported in that study, at least two of them were attributed to severe infections.

The resolution of the WBC lineage changes after treatment of CS is also important for the follow up and counseling of the patients. Although there are reports of continuous immune dysregulation, even after normalization of the cortisol levels, most of the previous studies have documented a rebound reactivation of the immune system, presenting for example with an increased incidence of autoimmune diseases $(35,36)$.

To our knowledge, this is the first study to investigate the WBC changes in pediatric patients with endogenous CS. One of the main strengths of the study is the large number of patients included, especially when considering the rare nature of the disease, since we serve as an international center for management of pediatric pituitary tumors. We were also able to follow most of the patients longitudinally after treatment, and thus document the improvement of the cells of the immune system after cure. A limitation of the study is the retrospective nature of the investigation. Thus, since the timing of the collection of the $\mathrm{CBC}$ at the time of diagnosis and at the follow-up was indicated by our protocol, it cannot be informative for the specific timing of the various WBC changes or their resolution. Furthermore, we relied on the review of the medical records to document the history of infections, which may be underestimated. Additionally, blood samples were not available to perform further diagnostic tests, such as lymphocyte phenotyping to detect the specific lymphocyte subtype changes, or evaluation of other inflammatory proteins and cytokines, in order to assess the changes of the secretory function of the immune cells. As it is known that the effect of GCs is not solely the effect on the number of the WBC cells, but also and, maybe even more importantly, on their function, further studies are required to describe these changes in pediatric endogenous CS.

In conclusion, pediatric patients with CS present with significant changes of the WBC counts, including decrease of the absolute lymphocyte count, which normalize after cure and return upon recurrence. These changes correlate with the morning and midnight cortisol levels and the UFC. The patients with lower lymphocyte count and higher cortisol levels, have higher risk for infections, and although most of the infections are mild, the possibility of a life-threatening infection cannot be excluded. These findings should be 


\section{WBC changes in Cushing syndrome}

considered as part of the phenotypic presentation of patients with CS and may serve as diagnostic clues when an obese patient is evaluated for an underlying pathology.

\section{STATEMENT OF FINANCIAL SUPPORT}

The work was supported by the Intramural Research Program, Eunice Kennedy Shriver National Institute of Child Health \& Human Development (NICHD), and the Clinical Center (CC), National Institutes of Health (NIH).

Disclosure: The authors declare no conflict of interest.

\section{REFERENCES}

1. Hench P. Effects of cortisone in the rheumatic diseases. Lancet 1950;2: 483-4.

2. Schappert SM, Rechtsteiner EA. Ambulatory medical care utilization estimates for 2006. Natl Health Stat Report 2008;8:1-29.

3. Oppong E, Cato AC. Effects of glucocorticoids in the immune system. Adv Exp Med Biol 2015;872:217-33.

4. Saffar AS, Ashdown H, Gounni AS. The molecular mechanisms of glucocorticoids-mediated neutrophil survival. Curr Drug Targets 2011;12: $556-62$.

5. Cox G. Glucocorticoid treatment inhibits apoptosis in human neutrophils. Separation of survival and activation outcomes. J Immunol 1995;154:4719-25.

6. Herold MJ, McPherson KG, Reichardt HM. Glucocorticoids in T cell apoptosis and function. Cell Mol Life Sci 2006;63:60-72.

7. Flammer JR, Rogatsky I. Minireview: glucocorticoids in autoimmunity: unexpected targets and mechanisms. Mol Endocrinol 2011;25:1075-86.

8. Masri-Iraqi H, Robenshtok E, Tzvetov G, Manistersky Y, Shimon I. Elevated white blood cell counts in Cushing's disease: association with hypercortisolism. Pituitary 2014;17:436-40.

9. Dimopoulos MA, Fernandez JF, Samaan NA, Holoye PY, VassilopoulouSellin R. Paraneoplastic Cushing's syndrome as an adverse prognostic factor in patients who die early with small cell lung cancer. Cancer 1992;69:66-71.

10. Nieman LK, Biller BM, Findling JW, et al. Treatment of Cushing's syndrome: an Endocrine Society Clinical Practice Guideline. J Clin Endocrinol Metab 2015;100:2807-31.

11. Stratakis CA. Diagnosis and clinical genetics of cushing syndrome in pediatrics. Endocrinol Metab Clin North Am 2016;45:311-28.

12. Karageorgiadis AS, Papadakis GZ, Biro J, et al. Ectopic adrenocorticotropic hormone and corticotropin-releasing hormone cosecreting tumors in children and adolescents causing cushing syndrome: a diagnostic dilemma and how to solve it. J Clin Endocrinol Metab 2015;100:141-8.

13. Baris HE, Baris S, Karakoc-Aydiner E, et al. The effect of systemic corticosteroids on the innate and adaptive immune system in children with steroid responsive nephrotic syndrome. Eur J Pediatr 2016;175: 685-93.

14. Serra-Bonett N, Al Snih S, Rodriguez MA. Effect of low-dose prednisone on leukocyte counts and subpopulations in patients with rheumatoid arthritis. J Clin Rheumatol 2009;15:148-9.

15. Youssef P, Roberts-Thomson P, Ahern M, Smith M. Pulse methylprednisolone in rheumatoid arthritis: effects on peripheral blood and synovial fluid neutrophil surface phenotype. J Rheumatol 1995;22:2065-71.

16. Ma H, Zhao L, Jiang Z, Jiang Y, Feng L, Ye Z. Dynamic changes in the numbers of different subsets of peripheral blood NK cells in patients with systemic lupus erythematosus following classic therapy. Clin Rheumatol 2014;33:1603-10.
17. De La Balze FA, Reifenstein EC Jr, Albright F. Differential blood counts in certain adrenal cortical disorders (Cushing's syndrome, Addison's disease and panhypopituitarism). J Clin Endocrinol Metab 1946;6:312-9.

18. Kronfol Z, Starkman M, Schteingart DE, Singh V, Zhang Q, Hill E. Immune regulation in Cushing's syndrome: relationship to hypothalamicpituitary-adrenal axis hormones. Psychoneuroendocrinology 1996;21: 599-608.

19. Masera RG, Staurenghi A, Sartori ML, Angeli A. Natural killer cell activity in the peripheral blood of patients with Cushing's syndrome. Eur J Endocrinol 1999;140:299-306.

20. Kumar S, Kelly AS. Review of childhood obesity: from epidemiology, etiology, and comorbidities to clinical assessment and treatment. Mayo Clin Proc 2017;92:251-65.

21. Keil MF. Quality of life and other outcomes in children treated for Cushing syndrome. J Clin Endocrinol Metab 2013;98:2667-78.

22. Albuquerque EV, Scalco RC, Jorge AA. MANAGEMENT OF ENDOCRINE DISEASE: Diagnostic and therapeutic approach of tall stature. Eur J Endocrinol 2017;176:R339-53.

23. Stratakis CA, Mastorakos G, Mitsiades NS, Mitsiades CS, Chrousos GP. Skin manifestations of Cushing disease in children and adolescents before and after the resolution of hypercortisolemia. Pediatr Dermatol 1998;15: 253-8.

24. Libuit LG, Karageorgiadis AS, Sinaii N, et al. A gender-dependent analysis of Cushing's disease in childhood: pre- and postoperative follow-up. Clin Endocrinol (Oxf) 2015;83:72-7.

25. Lodish MB, Hsiao HP, Serbis A, et al. Effects of Cushing disease on bone mineral density in a pediatric population. J Pediatr 2010;156:1001-5.

26. Fan PT, Yu DT, Clements PJ, Fowlston S, Eisman J, Bluestone R. Effect of corticosteroids on the human immune response: comparison of one and three daily $1 \mathrm{gm}$ intravenous pulses of methylprednisolone. J Lab Clin Med 1978;91:625-34.

27. Sapolsky RM, Romero LM, Munck AU. How do glucocorticoids influence stress responses? Integrating permissive, suppressive, stimulatory, and preparative actions. Endocr Rev 2000;21:55-89.

28. Fareau GG, Vassilopoulou-Sellin R. Hypercortisolemia and infection. Infect Dis Clin North Am 2007;21:639-57, viii.

29. Stuck AE, Minder CE, Frey FJ. Risk of infectious complications in patients taking glucocorticosteroids. Rev Infect Dis 1989;11:954-63.

30. Plotz CM, Knowlton AI, Ragan C. The natural history of Cushing's syndrome. Am J Med 1952;13:597-614.

31. Ejaz S, Vassilopoulou-Sellin R, Busaidy NL, et al. Cushing syndrome secondary to ectopic adrenocorticotropic hormone secretion: the University of Texas MD Anderson Cancer Center Experience. Cancer 2011;117:4381-9.

32. Sarlis NJ, Chanock SJ, Nieman LK. Cortisolemic indices predict severe infections in Cushing syndrome due to ectopic production of adrenocorticotropin. J Clin Endocrinol Metab 2000;85:42-7.

33. Ilias I, Torpy DJ, Pacak K, Mullen N, Wesley RA, Nieman LK. Cushing's syndrome due to ectopic corticotropin secretion: twenty years' experience at the National Institutes of Health. J Clin Endocrinol Metab 2005;90: 4955-62.

34. Gkourogianni A, Lodish MB, Zilbermint M, et al. Death in pediatric Cushing syndrome is uncommon but still occurs. Eur J Pediatr 2015;174: 501-7.

35. Bartz SK, Karaviti LP, Brandt ML, et al. Residual manifestations of hypercortisolemia following surgical treatment in a patient with Cushing syndrome. Int J Pediatr Endocrinol 2015 2015;19.

36. da Mota F, Murray C, Ezzat S. Overt immune dysfunction after Cushing's syndrome remission: a consecutive case series and review of the literature. J Clin Endocrinol Metab 2011;96:E1670-4. 